\title{
Impaired atrial synchronicity in patients with metabolic syndrome associated with insulin resistance and independent of hypertension
}

\author{
Shao-Hua Li ${ }^{1}$, Bo Yang ${ }^{1}$, Hui-Ping Gong, Hong-Wei Tan, Ming Zhong, Yun Zhang and Wei Zhang
}

The risk of developing atrial fibrillation is increased in patients with metabolic syndrome, but atrial conduction properties are uncharacterized in patients who have metabolic syndrome without atrial arrhythmia. We used tissue Doppler imaging to evaluate intra- and interatrial synchronicity in such patients. The imaging was performed in 145 patients with metabolic syndrome and 110 controls. Atrial synchronicity was determined from the intervals between the onset of the P-wave to the onset of the A-wave at the left atrial free wall (P-LA), interatrial septum (P-IAS) and right atrial free wall (P-RA). Intra-atrial synchronicity was defined as the differences between P-IAS and P-RA (RA synchronicity) and between P-LA and P-IAS (LA synchronicity). Interatrial synchronicity was defined as the difference between P-LA and P-RA. P-LA and P-IAS were significantly prolonged in the metabolic syndrome group relative to the control group (P-LA: $64.34 \pm 13.99$ vs. $55.35 \pm 12.67, P<0.001$; $P$-IAS: $36.49 \pm 12.39$ vs. $31.55 \pm 11.61, P=0.001$ ), whereas $P-R A$ showed no difference. As a result, this caused impaired intra- and interatrial synchronicity in patients with metabolic syndrome. Stepwise multivariate linear analysis revealed insulin resistance as an independent predictor of impaired intra- and interatrial synchronicity. Subgroup analysis indicated that there was no difference in atrial asynchrony between non-hypertensive and hypertensive subgroups in metabolic syndrome patients. In conclusion, patients with metabolic syndrome without atrial arrhythmia have impaired intra- and interatrial synchronicity. Insulin resistance has an important role in impaired atrial conduction in these patients.

Hypertension Research (2009) 32, 791-796; doi:10.1038/hr.2009.105; published online 17 July 2009

Keywords: insulin resistance; metabolic syndrome; synchronicity; tissue Doppler imaging

\section{INTRODUCTION}

Atrial remodeling has been observed in many cardiovascular diseases, including hypertension, ${ }^{1}$ myocardial infarction ${ }^{2}$ and heart failure. $^{3}$ Recent studies ${ }^{4,5}$ have shown that metabolic syndrome can increase the risk for the development of atrial fibrillation (AF); this, in turn, indicates that metabolic syndrome may also lead to electrical and structural remodeling of the atria. However, to date, there have been no direct data revealing whether atrial conduction is impaired in patients with metabolic syndrome without atrial arrhythmia.

As hypertension is an important factor in atrial remodeling, and other components of metabolic syndrome, including obesity, impaired glucose tolerance and dyslipidemia, are all associated with the development of $\mathrm{AF}^{6-8}$ we hypothesize that atrial conduction should be impaired in metabolic syndrome patients without atrial arrhythmia. In addition, we sought to clarify whether hypertension is the main factor that affects atrial conduction in metabolic syndrome as well as to determine the contributions of other components of metabolic syndrome to atrial conduction.
The noninvasive determination of atrial conduction properties can be performed by measuring delayed intra- and interatrial conduction using color tissue Doppler imaging, which has been proved to be useful for evaluating intra- and interatrial synchronicity in patients with heart failure. ${ }^{9}$

In this study, we used color tissue Doppler imaging to assess intraand interatrial synchronicity in patients with metabolic syndrome without atrial arrhythmia. In addition, we analyzed each component of metabolic syndrome that influences atrial synchronicity and determined the key factor among them.

\section{METHODS}

Study population

We selected 145 consecutive ambulatory patients with metabolic syndrome who visited our hospital from October 2006 to August 2007; some of these subjects were also used to investigate the left ventricular synchronicity in metabolic syndrome. ${ }^{10}$ Inclusion criteria, in accordance with the International Diabetes Federation definition of metabolic syndrome, were as follows: ${ }^{11}$

Department of Cardiology, Key Laboratory of Cardiovascular Remodeling and Function Research, Chinese Ministry of Education and Chinese Ministry of Public Health, QiLu Hospital, Shandong University, Jinan, China

Correspondence: Dr W Zhang, Department of Cardiology, Key Laboratory of Cardiovascular Remodeling and Function Research, Chinese Ministry of Education and Chinese Ministry of Public Health, QiLu Hospital, Shandong University, no. 107 West Wenhua Road, Jinan 250012, China.

E-mail: zhangwei7@medmail.com.cn

${ }^{1}$ These authors contributed equally to this work and should be considered co-first authors.

Received 4 March 2009; revised 17 May 2009; accepted 2 June 2009; published online 17 July 2009 
(1) central obesity, defined as waist circumference $\geqslant 90 \mathrm{~cm}$ for Chinese men and $\geqslant 80 \mathrm{~cm}$ for Chinese women and (2) any two of the following four factors: (a) triglyceride level $>150 \mathrm{mg}$ per $100 \mathrm{ml}\left(1.7 \mathrm{mmoll}^{-1}\right)$, or specific treatment for this lipid abnormality; (b) high-density lipoprotein cholesterol (HDL-C) $<40 \mathrm{mg}$ per $100 \mathrm{ml}\left(0.9 \mathrm{mmoll}^{-1}\right)$ in male patients and $<50 \mathrm{mg}$ per $100 \mathrm{ml}$ $\left(1.1 \mathrm{mmoll}^{-1}\right)$ in female patients, or specific treatment for this lipid abnormality; (c) systolic blood pressure $\geqslant 130$ or diastolic blood pressure $\geqslant 85 \mathrm{~mm} \mathrm{Hg}$, or treatment of previously diagnosed hypertension; or (d) fasting plasma glucose $\geqslant 100 \mathrm{mg}$ per $100 \mathrm{ml}\left(5.6 \mathrm{mmoll}^{-1}\right)$, or previously diagnosed type II diabetes. Patients with left or right bundle branch block, atrioventricular block, atrial fibrillation, atrial flutter, ventricular arrhythmia or structural heart disease were excluded from the study. Standard electrocardiography criteria were used to diagnose atrial fibrillation ${ }^{12}$ and atrial flutter, ${ }^{13}$ consistent with previous studies. The control group consisted of 110 healthy subjects, enrolled through routine physical examinations in our hospital, who had no history of cardiac disease, cardiac arrhythmia or known structural heart disease.

All subjects in both the metabolic syndrome group and the control group underwent complete cardiovascular evaluation, as described earlier. ${ }^{10}$ Briefly, after an 8-h fast, the subjects received a physical examination including the assessment of heart rate, blood pressure, and levels of fasting glucose, triglycerides, total cholesterol, low-density lipoprotein cholesterol and HDL-C. They also underwent 12-lead electrocardiography, 24-h Holter evaluation and comprehensive 2-D and Doppler echocardiography. Serum insulin levels were determined using a radio-immunoassay kit (Dongya Ltd., Beijing, China), and insulin resistance was assessed from fasting glucose and fasting insulin concentrations using the Homeostasis Model Assessment (HOMA) (fasting insulin in microunits per milliliter multiplied by fasting glucose in millimoles per liter divided by 22.5). ${ }^{14}$

Informed consent was obtained from all subjects following a protocol approved by the Ethics Committee of QiLu Hospital, Shandong University. The study was conducted in accordance with the Declaration of Helsinki.

\section{Two-dimensional and M-mode echocardiography}

The method used in this study was described earlier. ${ }^{10}$ In brief, transthoracic echocardiography was performed with an ultrasonographic system equipped with a 2.5- to 3.5-MHz transducer (Vivid 7, GE Vingmed Ultrasound AS, Horten, Norway). One technician operated the machine and had no previous information about the study. The LV diameter in end diastole, interventricular septum (IVSd) and LV posterior wall thickness in end diastole were measured from the M-mode readings. LV mass (LVM) was calculated by the formula of Devereux ${ }^{15}$ and was indexed to the allometric power of height ( $\left.\mathrm{LVM} / \mathrm{Ht}^{2.7}\right)$.The LV ejection fraction was estimated by the use of a modified version of Simpson's biplane method.

\section{Tissue doppler echocardiography}

Tissue Doppler imaging was performed to assess the atrial conduction properties using the apical 4-chamber view. ${ }^{9}$ Gain settings, filters and pulse repetitive frequency were adjusted to optimize the color saturation. The left and right atria were recorded simultaneously, with a minimal frame rate of 96 frames per second. At least three consecutive beats were stored and analyzed off-line. Right atrial (RA) and left atrial (LA) surfaces were established by tracing the atria at the mid-diastole in the 2-dimensional apical 4-chamber view. The P-wave duration was measured in lead II during standard electrocardiography and was averaged over 10 consecutive beats.

To assess the synchronicity of atria, myocardial velocity curves were reconstituted off-line below the tricuspid and mitral annulus on the RA free wall, the interatrial septum and the LA free wall. The time from the onset of the $\mathrm{P}$-wave to the onset of the A-wave at the right atrium (P-RA), the interatrial septum (P-IAS) and the left atrium (P-LA) was measured. Intra-atrial synchronicity was defined as the differences between P-IAS and P-RA (RA synchronicity) and between P-LA and P-IAS (LA synchronicity). Interatrial synchronicity was defined as the difference between P-LA and P-RA (Figure 1).

The interobserver and intraobserver variabilities for measuring synchronicity were compared over 60 consecutive measurements and were 5.3 and $4.7 \%$, respectively, which are similar to the variabilities in earlier studies. ${ }^{10}$

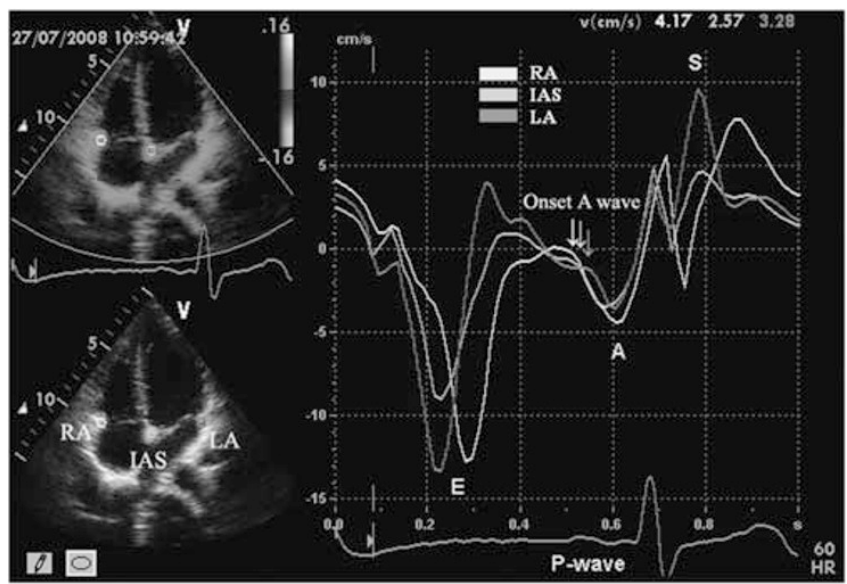

Figure 1 Tissue doppler myocardial velocity curves were obtained from the right atrium (RA) (yellow), the interatrial septum (IAS) (green) and the left atrium (LA) (red), just below the level of the tricuspid and mitral annulus. Arrows, the onset of A waves. A full color version of this figure is available at the Hypertension Research journal online.

\section{Statistical analysis}

Data analysis involved the use of the Statistical Package for the Social Sciences for Windows, version 13.0 (SPSS Inc., Chicago, IL, USA). Data are expressed as mean \pm s.d. Student's two-tailed $t$-test and $\chi^{2}$-test were used as appropriate. Pearson's correlation was used to assess the correlation between intra- and interatrial synchronicity and several potential factors, including age, blood pressure, waist-to-hip ratio, body mass index, HDL-C, fasting blood glucose, $\mathrm{LVM} / \mathrm{Ht}^{2.7}$ and HOMA index. Stepwise multiple variable linear regressions ${ }^{16}$ were used to determine the variables for predicting intra- and interatrial synchronicity. Entry into the regression model required a $P<0.10$. A $P<0.05$ was considered statistically significant.

\section{RESULTS}

A total of 110 subjects in the control group and 145 in the metabolic syndrome group were included in our study. There were no statistical differences in age and gender, but in the metabolic syndrome group, weight, body mass index, waist-to-hip ratio, systolic and diastolic blood pressure and the levels of triglycerides and low-density lipoprotein cholesterol were significantly increased. Moreover, compared with the control group, insulin and the HOMA index were also increased, whereas the HDL-C level was reduced significantly (Table 1).

IVSd, LV posterior wall thickness in end diastole and $\mathrm{LVM} / \mathrm{Ht}^{2.7}$ were significantly higher in the metabolic syndrome group than in controls, but the LV ejection fraction and LA and RA surfaces did not differ (Table 2). The P-LA and P-IAS were significantly prolonged in the metabolic syndrome group compared with the control group (P-LA: $64.34 \pm 13.99$ vs. $55.35 \pm 12.67, P<0.001$; P-IAS: $36.49 \pm 12.39$ vs. $31.55 \pm 11.61, P=0.001)$. However, the $\mathrm{P}-\mathrm{RA}$ was not significantly prolonged, which resulted in impaired intra- and interatrial synchronicity in the patients with metabolic syndrome (Table 2).

In univariate analysis with Pearson's correlation, RA synchronicity was associated with the HOMA index and HDL-C in patients with metabolic syndrome, LA synchronicity was associated with the HOMA index and fasting blood glucose and interatrial synchronicity was correlated with the HOMA index. However, stepwise multivariate analysis revealed only the HOMA index to be an independent predictor of RA, LA and interatrial synchronicity (Table 3 ).

As hypertension is an important factor in atrial remodeling, the impaired intra- and interatrial synchronicity may be ascribed to the influence of hypertension. To clarify the contribution of hypertension 
Table 1 Clinical characteristics of controls and patients with metabolic syndrome

\begin{tabular}{|c|c|c|c|}
\hline & $\begin{array}{l}\text { Control group } \\
\qquad(\mathrm{n}=110)\end{array}$ & $\begin{array}{l}\text { MS group } \\
(\mathrm{n}=145)\end{array}$ & P-value \\
\hline Age (years) & $54.71 \pm 8.05$ & $56.94 \pm 9.79$ & 0.063 \\
\hline Female (\%) & $71(64)$ & $84(58)$ & 0.066 \\
\hline \multicolumn{4}{|l|}{ Clinical features } \\
\hline Weight (kg) & $65.41 \pm 9.53$ & $76.95 \pm 13.37$ & $<0.001$ \\
\hline Height (cm) & $162.98 \pm 6.53$ & $164.15 \pm 8.05$ & 0.202 \\
\hline $\mathrm{BMI}\left(\mathrm{kg} \mathrm{m}^{-2}\right)$ & $24.56 \pm 2.76$ & $28.46 \pm 3.64$ & $<0.001$ \\
\hline Waist circumference $(\mathrm{cm})$ & $84.68 \pm 8.19$ & $96.92 \pm 9.61$ & $<0.001$ \\
\hline Hip circumference (cm) & $98.26 \pm 6.41$ & $106.31 \pm 7.95$ & $<0.001$ \\
\hline Waist-to-hip ratio & $0.87 \pm 0.04$ & $0.94 \pm 0.07$ & $<0.001$ \\
\hline Systolic BP (mm Hg) & $116.51 \pm 10.44$ & $151.09 \pm 21.04$ & $<0.001$ \\
\hline Diastolic BP (mm Hg) & $75.56 \pm 6.47$ & $93.41 \pm 13.69$ & $<0.001$ \\
\hline Heart rate (b.p.m.) & $69 \pm 9$ & $70 \pm 10$ & 0.237 \\
\hline $\mathrm{P}$ duration (ms) & $113 \pm 8$ & $115 \pm 10$ & 0.193 \\
\hline Total cholesterol $\left(\mathrm{mmol} \mathrm{I}^{-1}\right)$ & $4.77 \pm 0.83$ & $5.51 \pm 1.07$ & $<0.001$ \\
\hline Triglycerides (mmol $\mathrm{I}^{-1}$ ) & $1.16 \pm 0.55$ & $2.43 \pm 2.18$ & $<0.001$ \\
\hline LDL cholesterol ( $\left.\mathrm{mmol}^{-1}\right)$ & $3.04 \pm 0.73$ & $3.70 \pm 0.97$ & $<0.001$ \\
\hline HDL cholesterol $\left(\mathrm{mmol} \mathrm{I}^{-1}\right)$ & $1.57 \pm 0.31$ & $1.27 \pm 0.32$ & $<0.001$ \\
\hline Fasting blood glucose (mmol $\left.\mathrm{I}^{-1}\right)$ & $4.86 \pm 0.60$ & $6.65 \pm 2.58$ & $<0.001$ \\
\hline Insulin $\left(\mu \cup \mathrm{ml}^{-1}\right)$ & $10.46 \pm 4.74$ & $19.81 \pm 10.55$ & $<0.001$ \\
\hline HOMA index & $2.31 \pm 1.24$ & $6.01 \pm 4.48$ & $<0.001$ \\
\hline \multicolumn{4}{|l|}{ Past diseases (n, \%) } \\
\hline Hypertension & $0(0)$ & $31(21)$ & - \\
\hline Type II diabetes mellitus & $0(0)$ & $38(26)$ & - \\
\hline \multicolumn{4}{|l|}{ Past medical history ( $n, \%)$} \\
\hline Beta-blockers & $0(0)$ & $3(2)$ & - \\
\hline Calcium antagonists & $0(0)$ & $8(5)$ & - \\
\hline ACEI or ARB & $0(0)$ & $2(1)$ & - \\
\hline Loop diuretics & $0(0)$ & $4(3)$ & - \\
\hline Oral hypoglycemia & $0(0)$ & $27(19)$ & - \\
\hline
\end{tabular}

Abbreviations: ACEI, angiotensin-converting enzyme inhibitor; ARB, angiotensin receptor blockers; BP, blood pressure; BMI, body mass index; HDL-C, high-density lipoprotein cholesterol; HOMA, the homeostasis model assessment; LDL-C, low-density lipoprotein cholesterol; MS, metabolic syndrome.

to the impaired atrial synchronicity, we divided the metabolic syndrome group into a non-hypertensive subgroup $(n=55)$ and a hypertensive subgroup $(n=73)$. Beta blockers, ${ }^{17}$ angiotensin-converting enzyme inhibitors and angiotensin receptor blockers ${ }^{18}$ have been thought to improve atrial electrical remodeling. Therefore, to avoid affecting the results, 17 patients using antihypertensive drugs such as beta blockers, angiotensin-converting enzyme inhibitors or angiotensin receptor blockers, calcium antagonists or diuretics were excluded from the subgroup analysis. Although the comparison of two-dimensional and M-mode echocardiographic parameters between the two subgroups showed that IVSd and $\mathrm{LVM} / \mathrm{Ht}^{2.7}$ were significantly increased in the hypertensive subgroup, no significant changes were observed between the two subgroups among the atrial indexes, including RA and LA size and RA, LA and interatrial synchronicity (Table 4).

Furthermore, to ascertain associations between impaired atrial synchronicity and atrial size, we divided the patients with metabolic syndrome into subgroups to reveal these relations (Table 5). The category criterion of right synchronicity was based on the upper two s.d. of the mean of the control group and, according to this criterion,
Table 2 Two-dimensional, M-mode and color tissue Doppler imaging echocardiography parameters for controls and patients with metabolic syndrome

\begin{tabular}{lccc}
\hline & $\begin{array}{c}\text { Control group } \\
(\mathrm{n}=110)\end{array}$ & $\begin{array}{c}\text { MS group } \\
(\mathrm{n}=145)\end{array}$ & P-value \\
\hline IVSd $(\mathrm{mm})$ & $9.83 \pm 1.04$ & $11.70 \pm 1.40$ & $<0.001$ \\
LVPWd (mm) & $8.96 \pm 1.01$ & $10.72 \pm 2.72$ & $<0.001$ \\
LVDd (mm) & $42.28 \pm 4.22$ & $43.86 \pm 5.39$ & $<0.05$ \\
LVM/Ht $2.7\left(\mathrm{~g} / \mathrm{m}^{2.7}\right)$ & $34.16 \pm 8.97$ & $46.11 \pm 10.84$ & $<0.001$ \\
LVEF $(\%)$ & $65.45 \pm 5.66$ & $66.24 \pm 6.78$ & 0.102 \\
RA surface $\left(\mathrm{cm}^{2}\right)$ & $14.87 \pm 3.23$ & $15.79 \pm 3.53$ & 0.164 \\
LA surface $\left(\mathrm{cm}^{2}\right)$ & $15.17 \pm 2.74$ & $17.53 \pm 3.91$ & 0.073 \\
P-RA (ms) & $22.74 \pm 8.17$ & $22.99 \pm 7.90$ & 0.802 \\
P-IAS (ms) & $31.55 \pm 11.61$ & $36.49 \pm 12.39$ & 0.001 \\
P-LA (ms) & $55.35 \pm 12.67$ & $64.34 \pm 13.99$ & $<0.001$ \\
RA synchronicity (ms) & $8.81 \pm 8.25$ & $13.50 \pm 10.12$ & $<0.001$ \\
LA synchronicity (ms) & $23.81 \pm 9.29$ & $27.85 \pm 13.25$ & 0.005 \\
Interatrial synchronicity (ms) & $32.61 \pm 10.68$ & $41.35 \pm 13.08$ & $<0.001$ \\
\hline
\end{tabular}

Abbreviations: IVSd, interventricular septum thickness; LVPWd, left ventricular posterior wall thickness in end diastole; LVDd, left ventricular diameter in end diastole; LVM/Ht2.7, left ventricular mass for height to the power 2.7; LVEF, left ventricular ejection fraction; LA, left atrial; RA, right atrial; P-RA, the time difference from the onset of the $P$ wave to the onset of the $A$ wave at the right atrium; P-IAS, the time difference from the onset of the $P$ wave to the onset of the A wave at the interatrial septum; P-LA, the time difference from the onset of the $P$ wave to the onset of the A wave at the left atrium; MS, metabolic syndrome.

patients with metabolic syndrome were divided into two subgroups (subgroup $<25.31 \mathrm{~ms}$ and subgroup $\geqslant 25.31 \mathrm{~ms}$ ). Similarly, category criteria for left and interatrial synchronicity in patients with metabolic syndrome were also based on the upper two s.d. of the mean of the control group. The results showed that between the two subgroups of LA synchronicity, a significant difference in the LA size could be observed. Moreover, significant differences in the LA size could be found between the two subgroups of interatrial synchronicity. Although significant differences in the RA size could not be detected between the two subgroups of RA synchronicity and interatrial synchronicity, a significant trend could still be found in subgroups of RA synchronicity $(P=0.063)$. Table 5 indicates that in patients with metabolic syndrome, the association between prolonged synchronicity and atrial size was more evident in the left atrium.

\section{DISCUSSION}

In this study, we showed that intra- and interatrial synchronicity were impaired in patients with metabolic syndrome without atrial arrhythmia, compared with the control subjects. In addition, the relationship between prolonged synchronicity and atrial size was more obvious in the left atrium. Furthermore, we found that the impaired synchronicity was significantly associated with insulin resistance but was not correlated with systolic and diastolic blood pressure in patients with metabolic syndrome, indicating that insulin resistance but not hypertension has an important role in the impaired atrial conduction in these patients.

It is well known that metabolic syndrome consists of obesity, hypertension, impaired glucose tolerance, hyperinsulinemia and dyslipidemia. ${ }^{19}$ Wang et al. ${ }^{6}$ have shown that obesity can increase the risk of new-onset AF. Meanwhile, the morbidity of AF is higher in patients with hypertension, ${ }^{20}$ and earlier studies have shown that type II diabetes mellitus is an independent predictor of $\mathrm{AF}^{21}$ Recent studies have further shown that metabolic syndrome not only increases the risk for the development of $\mathrm{AF}^{4,5}$ but also increases the risk of recurrence of AF after catheter ablation. ${ }^{22}$ Consequently, this indicates 
Table 3 Univariate and stepwise multivariate linear regression analysis of impaired intra- and interatrial synchronicity for each component of the metabolic syndrome

\begin{tabular}{|c|c|c|c|c|c|c|c|c|c|}
\hline & \multicolumn{3}{|c|}{ RA asynchrony } & \multicolumn{3}{|c|}{ LA asynchrony } & \multicolumn{3}{|c|}{ Interatrial asynchrony } \\
\hline & \multicolumn{2}{|c|}{ Univariate } & $\begin{array}{c}\text { Multivariate } \\
\mathrm{P}\end{array}$ & \multicolumn{2}{|c|}{ Univariate } & $\begin{array}{c}\text { Multivariate } \\
\mathrm{P}\end{array}$ & \multicolumn{2}{|c|}{ Univariate } & $\begin{array}{c}\text { Multivariate } \\
\mathrm{P}\end{array}$ \\
\hline Age & 0.081 & 0.208 & NS & 0.046 & 0.321 & NS & 0.114 & 0.124 & NS \\
\hline BMI & 0.091 & 0.179 & NS & 0.013 & 0.448 & NS & 0.062 & 0.266 & NS \\
\hline Waist-to-hip ratio & 0.020 & 0.421 & NS & 0.098 & 0.162 & NS & 0.084 & 0.197 & NS \\
\hline $\mathrm{HDL}-\mathrm{C}$ & -0.138 & $0.049 *$ & NS & -0.095 & 0.170 & NS & -0.016 & 0.434 & NS \\
\hline FBG & 0.121 & 0.052 & NS & 0.125 & $0.047^{*}$ & NS & 0.035 & 0.363 & NS \\
\hline $\mathrm{LVM} / \mathrm{Ht}^{2.7}$ & 0.042 & 0.338 & NS & 0.083 & 0.202 & NS & 0.051 & 0.304 & NS \\
\hline HOMA index & 0.167 & $0.037 *$ & $0.040^{*}$ & 0.178 & $0.036^{*}$ & $0.036^{*}$ & 0.170 & $0.043^{*}$ & $0.044^{*}$ \\
\hline
\end{tabular}

Abbreviations: BMI, body mass index; BP, blood pressure; FBG, fasting blood glucose; HDL-C, high-density lipoprotein cholesterol; LVM/Ht2.7, left ventricular mass for height to the power 2.7; HOMA, homeostasis model assessment; RA, right atrial; $L A=$ left atrial. ${ }^{*} P<0.05$, statistically significant.

Table 4 Comparison of two-dimensional, M-mode and color tissue Doppler-imaging echocardiography parameters among patients with metabolic syndrome

\begin{tabular}{lccr}
\hline & $\begin{array}{c}\text { Non hypertensive } \\
\text { subgroup }(\mathrm{n}=55)\end{array}$ & $\begin{array}{c}\text { Hypertensive } \\
\text { subgroup }(\mathrm{n}=73)\end{array}$ & P-value \\
\hline IVSd $(\mathrm{mm})$ & $11.18 \pm 1.31$ & $12.08 \pm 1.38$ & 0.001 \\
LVPWd $(\mathrm{mm})$ & $10.23 \pm 1.04$ & $11.10 \pm 1.49$ & 0.134 \\
LVDd $(\mathrm{mm})$ & $43.41 \pm 4.13$ & $43.86 \pm 6.10$ & 0.679 \\
LVM/ $\mathrm{Ht}^{2.7}\left(\mathrm{~g} / \mathrm{m}^{2.7}\right)$ & $41.33 \pm 7.83$ & $49.14 \pm 11.12$ & $<0.001$ \\
LVEF $(\%)$ & $66.97 \pm 5.98$ & $66.14 \pm 6.52$ & 0.902 \\
RA surface $\left(\mathrm{cm}^{2}\right)$ & $15.43 \pm 3.37$ & $15.92 \pm 3.69$ & 0.442 \\
LA surface $\left(\mathrm{cm}^{2}\right)$ & $16.78 \pm 3.88$ & $17.97 \pm 4.23$ & 0.105 \\
P-RA (ms) & $21.77 \pm 4.82$ & $23.78 \pm 8.40$ & 0.099 \\
P-IAS (ms) & $35.42 \pm 11.75$ & $37.59 \pm 12.12$ & 0.348 \\
P-LA (ms) & $61.97 \pm 12.36$ & $65.16 \pm 14.54$ & 0.231 \\
RA synchronicity (ms) & $13.65 \pm 11.38$ & $13.81 \pm 9.92$ & 0.939 \\
LA synchronicity (ms) & $26.55 \pm 12.96$ & $27.57 \pm 12.58$ & 0.676 \\
Interatrial synchronicity (ms) & $40.21 \pm 13.10$ & $41.38 \pm 12.35$ & 0.628 \\
\hline
\end{tabular}

Abbreviations: IVSd, interventricular septum thickness; LVPWd, left ventricular posterior wall thickness in end diastole; LVDd, left ventricular diameter in end diastole; LVM/Ht ${ }^{2.7}$, left ventricular mass for height to the power 2.7; LVEF, left ventricular ejection fraction; LA, left atrial; RA, right atrial; P-RA, the time difference from the onset of the $\mathrm{P}$ wave to the onset of the $A$ wave at the right atrium; $P$-IAS, the time difference from the onset of the $P$ wave to the onset of the $A$ wave at the interatrial septum; $P$-LA, the time difference from the onset of the $P$ wave to the onset of the A wave at the left atrium.

that metabolic syndrome may result in electrical and structural remodeling of the atria. However, to date, no data have revealed atrial conduction properties in patients who have metabolic syndrome without atrial arrhythmia. In this study, we used color tissue Doppler imaging to detect intra- and interatrial synchronicity and found that P-LA and P-IAS were significantly prolonged in the metabolic syndrome group compared with the control group; as a result, the RA, LA and interatrial synchronicity were significantly different from those in the controls. This finding indicates that the intra- and interatrial conduction properties were impaired in patients with metabolic syndrome before developing atrial flutter or AF.

Although the mechanism of metabolic syndrome is still not well known, insulin resistance is widely considered to have an important role in its pathogenesis. ${ }^{23}$ The original conceptualization of this
Table 5 Comparison of right and left atrial size based on intra- and interatrial synchronicity in patients with metabolic syndrome

\begin{tabular}{|c|c|c|c|}
\hline & $R A$ surface $\left(\mathrm{cm}^{2}\right)$ & LA surface $\left(\mathrm{cm}^{2}\right)$ & $\mathrm{P}$-value \\
\hline \multicolumn{4}{|l|}{$R A$ synchronicity $(m s)^{a}$} \\
\hline$<25.31(n=117)$ & $15.52 \pm 3.42$ & NA & 0.063 \\
\hline$\geqslant 25.31(n=28)$ & $16.92 \pm 3.98$ & & \\
\hline \multicolumn{4}{|l|}{ LA synchronicity $(m s)^{b}$} \\
\hline$<42.39(n=115)$ & NA & $16.89 \pm 3.24$ & 0.024 \\
\hline$\geqslant 42.39(n=30)$ & & $19.98 \pm 5.74$ & \\
\hline \multicolumn{4}{|c|}{ Interatrial synchronicity $(m s)^{c}$} \\
\hline$<53.97(n=108)$ & $15.51 \pm 3.49$ & $16.77 \pm 3.31$ & $0.107^{\mathrm{d}} / 0.021^{\mathrm{e}}$ \\
\hline$\geqslant 53.97(n=37)$ & $16.61 \pm 3.75$ & $19.74 \pm 5.68$ & \\
\hline \multicolumn{4}{|c|}{$\begin{array}{l}\text { aThe category criterion of right synchronicity was based on the upper two s.d. of the mean of } \\
\text { the control group. } \\
\text { bThe category criterion of left synchronicity was based on the upper two s.d. of the mean of the } \\
\text { control group. } \\
\text { 'The category criterion of interatrial synchronicity was based on the upper two s.d. of the mean } \\
\text { of the control group. } \\
{ }^{d} \text { Comparison of right atrial size based on the subgroups of interatrial synchronicity. } \\
\text { eComparison of left atrial size based on the subgroups of interatrial synchronicity. } \\
\text { Abbreviations: RA, right atrial; LA, left atrial; NA, not applicable. }\end{array}$} \\
\hline
\end{tabular}

syndrome was based on the resistance to the metabolic actions of insulin. ${ }^{24}$ Meanwhile, insulin resistance can result in the activation of many proinflammatory transcription factors ${ }^{25}$ and induce the generation of reactive oxygen species. ${ }^{23,26}$ Inflammation and oxidative stress have been proposed as common etiological factors in the pathogenesis of $\mathrm{AF}^{27-29}$ Therefore, insulin resistance may contribute to atrial electrical remodeling in patients with metabolic syndrome. In this study, univariate analysis with Pearson's correlation showed that the HOMA index was significantly associated with impaired RA, LA and interatrial synchronicity in patients with metabolic syndrome; in addition, HDL-C was correlated with RA asynchrony, and fasting blood glucose was correlated with LA asynchrony. Furthermore, stepwise multivariate analysis revealed only the HOMA index as an independent predictor of impaired RA, LA and interatrial synchronicity, which indicates that insulin resistance has an important role in the impaired intra- and interatrial synchronicity. However, the exact 
mechanism of insulin resistance that results in impaired atrial synchronicity and atrial electrical remodeling is still not well understood. It may be related to an altered myocardial protein degradation, ${ }^{30}$ atrial myocardial fibrosis ${ }^{31}$ or disruption of cardiocyte coupling at gap junctions, ${ }^{32}$ which may finally cause electrophysiological instability in the atrial myocardium and lead to impaired atrial synchronicity and atrial electrical remodeling. Further studies are needed to identify in detail the exact mechanisms.

The fact that hypertension can induce atrial remodeling has been well accepted; ${ }^{1,20}$ in other words, abnormal hemodynamics has an important role in atrial structural and electrical remodeling. Given that hypertension is one of the components of metabolic syndrome, the impaired intra- and interatrial synchronicity may be ascribed mainly to hypertension. To clarify these associations, we divided the metabolic syndrome group into two subgroups: a non-hypertensive subgroup $(n=55)$ and a hypertensive subgroup $(n=73)$. Twodimensional and M-mode echocardiography showed that IVSd and $\mathrm{LVM} / \mathrm{Ht}^{2.7}$ were significantly increased, indicating that hypertension was, in fact, an important factor influencing the cardiac structure. However, in this study, no significant changes among the atrial indexes, including RA and LA size and RA, LA and interatrial synchronicity, were observed between the two subgroups. Moreover, the univariate analysis with Pearson's correlation and stepwise multivariate analysis also showed that neither systolic nor diastolic blood pressure was associated with impaired intra- and interatrial synchronicity. These results indicate that hemodynamics may not be the main factor in atrial electrical remodeling in patients with metabolic syndrome. Owing to the small number of subjects in this study, large-scale investigations are needed to confirm our conclusion.

Although impaired atrial synchronicity could be clearly observed between the metabolic syndrome group and the control group, no significant differences in the size of the right or left atria could be found between these two groups. As is well known, AF is often accompanied by enlarged atria or impaired atrial function. Therefore, we considered that among metabolic syndrome patients without atrial arrhythmia, those with severely impaired atrial synchronicity may have an enlarged atrium. Our present results showed that patients with metabolic syndrome and severely prolonged left atrial or interatrial synchronicity (greater than the upper two s.d. of the mean of the control group) had significantly enlarged left atria, which indicates that the influence of impaired atrial synchronicity on atrial size or function was more evident in the left atrium. However, this is the first time that this method has been used to detect the correlation between atrial synchronicity and atrial size, and further studies should be performed to investigate this issue more clearly.

In this study, the P-wave duration was determined, but there was no significant difference between the metabolic syndrome group and the control group in the duration, indicating that color tissue Doppler imaging may be more sensitive than P-wave duration to detect atrial conduction properties, which has been proved by earlier studies. ${ }^{9,33}$

Despite the intriguing findings of this study, certain limitations should be stressed. A recent study ${ }^{34}$ proposed that a single-tissue Doppler imaging test might not be sufficient to characterize intraventricular dyssynchrony, and therefore may also be insufficient to determine intra- and interatrial synchronicity in one test. Meanwhile, the number of patients with arterial hypertension receiving medical treatment was very low (only 17 patients) in the metabolic syndrome group, and most of them had not been diagnosed earlier with hypertension. Therefore, these patients might be in an early stage of hypertension, and abnormal hemodynamics may not deeply affect atrial remodeling. Further studies in patients with metabolic syndrome and severe hypertension should be performed to verify our results.

In conclusion, patients who have metabolic syndrome without atrial arrhythmia have impaired intra- and interatrial synchronicity, which indicates that these patients already have atrial electrical remodeling. Insulin resistance has an important role in impaired atrial conduction in these patients. Further studies should focus on the exact mechanisms and therapy of this electrical remodeling.

\section{CONFLICT OF INTEREST}

The authors declare no conflict of interest.

\section{ACKNOWLEDGEMENTS}

This work was supported by research grants from the National Natural Science Foundation of China (30670874, 30570748 and 30871038) and the National Basic Research Program of China (973 Program, Grant No.: 2009CB521904).

1 Miller JT, O'Rourke RA, Crawford MH. Left atrial enlargement: an early sign of hypertensive heart disease. Am Heart J 1988; 116: 1048-1051.

2 Beinart R, Boyko V, Schwammenthal E, Kuperstein R, Sagie A, Hod H, Matetzky S, Behar S, Eldar M, Feinberg MS. Long-term prognostic significance of left atrial volume in acute myocardial infarction. J Am Coll Cardiol 2004; 44: 327-334.

3 Meris A, Amigoni M, Uno H, Thune JJ, Verma A, Kober L, Bourgoun M, McMurray JJ, Velazquez EJ, Maggioni AP, Ghali J, Arnold JM, Zelenkofske S, Pfeffer MA, Solomon SD. Left atrial remodelling in patients with myocardial infarction complicated by heart failure, left ventricular dysfunction, or both: the VALIANT Echo study. Eur Heart $J$ 2009; 30: 56-65.

4 Watanabe H, Tanabe N, Watanabe T, Darbar D, Roden DM, Sasaki S, Aizawa Y. Metabolic syndrome and risk of development of atrial fibrillation: the Niigata preventive medicine study. Circulation 2008; 117: 1255-1260.

5 Nicolaou VN, Papadakis JE, Karatzis EN, Dermitzaki SI, Tsakiris AK, Skoufas PD. Impact of the metabolic syndrome on atrial size in patients with new-onset atrial fibrillation. Angiology 2007; 58: 21-25.

6 Wang TJ, Parise H, Levy D, D'Agostino Sr RB, Wolf PA, Vasan RS, Benjamin EJ. Obesity and the risk of new-onset atrial fibrillation. JAMA 2004; 292: 2471-2477.

7 Movahed MR, Hashemzadeh M, Jamal MM. Diabetes mellitus is a strong, independent risk for atrial fibrillation and flutter in addition to other cardiovascular disease. Int $\mathrm{J}$ Cardiol 2005; 105: 315-318.

8 Dublin S, French B, Glazer NL, Wiggins KL, Lumley T, Psaty BM, Smith NL, Heckbert SR. Risk of new-onset atrial fibrillation in relation to body mass index. Arch Intern Med 2006; 166: 2322-2328.

9 Van Beeumen K, Duytschaever M, Tavernier R, Van de Veire N, De Sutter J. Intra- and interatrial asynchrony in patients with heart failure. Am J Cardiol 2007; 99: 79-83.

10 Li SH, Yang B, Gong HP, Tan HW, Zhong M, Zhang Y, Zhang W. Impaired left ventricular synchronicity in patients with metabolic syndrome, regardless of hypertension. $J$ Hypertens 2009; 27: 869-875.

11 International Diabetes Federation. The IDF Consensus Worldwide Definition of the Metabolic Syndrome. International Diabetes Federation: Belgium, 2005.

12 Lip GY, Watson RD. ABC of atrial fibrillation. Differential diagnosis of atrial fibrillation. BMJ 1995; 311: 1495-1498.

13 Sawhney NS, Feld GK. Diagnosis and management of typical atrial flutter. Med Clin North Am 2008; 92: 65-85.

14 Matthews DR, Hosker JP, Rudenski AS, Naylor BA, Treacher DF, Turner RC. Homeostasis model assessment: insulin resistance and beta-cell function from fasting plasma glucose and insulin concentrations in man. Diabetologia 1985; 28: 412-419.

15 Devereux RB, Pickering TG, Alderman MH, Chien S, Borer JS, Laragh JH. Left ventricular hypertrophy in hypertension. Prevalence and relationship to pathophysiologic variables. Hypertension 1987; 9: II53-II60.

16 Harrell FE. Regression Modeling Strategies: With Applications to Linear Models, Logistic Regression, and Survival Analysis, 2nd edn. Springer: New York, 2006, pp 53-84.

17 Nasr IA, Bouzamondo A, Hulot JS, Dubourg O, Le Heuzey JY, Lechat P. Prevention of atrial fibrillation onset by beta-blocker treatment in heart failure: a meta-analysis. Eur Heart J 2007; 28: 457-462.

18 Makkar KM, Sanoski CA, Spinler SA. Role of angiotensin-converting enzyme inhibitors, angiotensin II receptor blockers, and aldosterone antagonists in the prevention of atrial and ventricular arrhythmias. Pacing Clin Electrophysiol 2003; 26: 436-439.

19 Haffner S, Taegtmeyer H. Epidemic obesity and the metabolic syndrome. Circulation 2003; 108: 1541-1545.

20 Ostgren CJ, Merlo J, Rastam L, Lindblad U. Atrial fibrillation and its association with type 2 diabetes and hypertension in a Swedish community. Diabetes Obes Metab 2004; 6: 367-374. 
21 Benjamin E, Levy D, Vaziri S, D’Agostino R, Belanger A, Wolf P. Independent risk factors for atrial fibrillation in a population based cohort. The Framingham Heart Study. JAMA 1994; 271: 840-844.

22 Tang RB, Dong JZ, Liu XP, Long DY, Yu RH, Kalifa J, Ma CS. Metabolic syndrome and risk of recurrence of atrial fibrillation after catheter ablation. Circ $J$ 2009; 73 : 438-443.

23 Dandona P, Aljada A, Chaudhuri A, Mohanty P, Garg R. Metabolic syndrome: a comprehensive perspective based on interactions between obesity, diabetes, and inflammation. Circulation 2005; 111: 1448-1454.

24 Reaven GM. Banting lecture 1988. Role of insulin resistance in human disease. Diabetes 1988; 37: 1595-1607.

25 Aljada A, Ghanim H, Mohanty P, Kapur N, Dandona P. Insulin inhibits the proinflammatory transcription factor early growth response gene-1 (Egr)-1 expression in mononuclear cells (MNC) and reduces plasma tissue factor (TF) and plasminogen activator inhibitor-1 (PAI-1) concentrations. J Clin Endocrinol Metab 2002; 87: 1419-1422.

26 Dandona P, Aljada A, Mohanty P, Ghanim H, Hamouda W, Assian E, Ahmad S. Insulin inhibits intranuclear nuclear factor kappaB and stimulates IkappaB in mononuclear cells in obese subjects: evidence for an anti-inflammatory effect? J Clin Endocrinol Metab 2001; 86: 3257-3265.

27 Chung MK, Martin DO, Sprecher D, Wazni O, Kanderian A, Carnes CA, Bauer JA, Tchou PJ, Niebauer MJ, Natale A, Van Wagoner DR. C-reactive protein elevation in patients with atrial arrhythmias: inflammatory mechanisms and persistence of atrial fibrillation. Circulation 2001; 104: 2886-2891.

28 Mihm MJ, Yu F, Carnes CA, Reiser PJ, McCarthy PM, Van Wagoner DR, Bauer JA. Impaired myofibrillar energetics and oxidative injury during human atrial fibrillation. Circulation 2001; 104: 174-180.

29 Kim YH, Lim DS, Lee JH, Shim WJ, Ro YM, Park GH, Becker KG, Cho-Chung YS, Kim MK. Gene expression profiling of oxidative stress on atrial fibrillation in humans. Exp Mol Med 2003; 35: 336-349.

30 Young ME, McNulty P, Taegtmeyer $\mathrm{H}$. Adaptation and maladaptation of the heart in diabetes: part 2: potential mechanisms. Circulation 2002; 105: 1861-1870.

31 Everett IV TH, Olgin JE. Atrial fibrosis and the mechanisms of atrial fibrillation. Heart Rhythm 2007; 4: S24-S27.

32 Polontchouk L, Haefliger JA, Ebelt B, Schaefer T, Stuhlmann D, Mehlhorn U, KuhnRegnier F, De Vivie ER, Dhein S. Effects of chronic atrial fibrillation on gap junction distribution in human and rat atria. J Am Coll Cardiol 2001; 38: 883-891.

33 Merckx K, De Vos C, Palmans A, Habets J, Cheriex E, Crijns H, Tieleman R. Atrial activation time determined by transthoracic Doppler tissue imaging can be used as an estimate of the total duration of atrial activation. J Am Soc Echocardiogr 2005; 18 : 940-944.

34 Vesely MR, Li S, Kop WJ, Reese A, Marshall J, Shorofsky SR, Gottlieb SS, Mehra MR, Gottdiener JS. Test-retest reliability of assessment for intraventricular dyssynchrony by tissue Doppler imaging echocardiography. Am J Cardiol 2008; 101: 645-650. 